\title{
Cannabinoid receptor activation reverses kainate-induced synchronized population burst firing in rat hippocampus
}

\author{
Rob Mason ${ }^{1}$ and Joseph F. Cheer ${ }^{2 *}$ \\ ' School of Biomedical Sciences, University of Nottingham Medical School, Queen's Medical Centre, Nottingham, UK \\ Department of Anatomy and Neurobiology, University of Maryland School of Medicine, Baltimore, MD, USA
}

Edited by:

Rui M. Costa, Champalimaud

Neuroscience Programme, Instituto

Gulbenkian de Ciência, Oeiras, Portugal

Reviewed by:

David M. Lovinger, National Institute on Alcohol Abuse and Alcoholism, NIH,

Bethesda, MD, USA

Henry H. Yin, Duke University, Durham, NC, USA

\section{*Correspondence:}

Joseph F. Cheer, Department of

Anatomy and Neurobiology, University

of Maryland School of Medicine, 20

Penn Street, HSF I, Baltimore, MD

21201, USA.

e-mail: jchee001@umaryland.edu
Cannabinoids have been shown to possess anticonvulsant properties in whole animal models of epilepsy. The present investigation sought to examine the effects of cannabinoid receptor activation on kainic acid (KA)-induced epileptiform neuronal excitability. Under urethane anesthesia, acute KA treatment (10 $\mathrm{mg} \mathrm{kg}^{-1}$, i.p.) entrained the spiking mode of simultaneously recorded neurons from random firing to synchronous bursting (\% change in burst rate). Injection of the high-affinity cannabinoid agonist (-)-11-hydroxy-8-tetrahydrocannabinol-dimethyl-heptyl (HU210, $100 \mu \mathrm{g} \mathrm{kg}^{-1}$, i.p.) following KA markedly reduced the burst frequency (\% decrease in burst frequency) and reversed synchronized firing patterns back to baseline levels. Pre-treatment with the central cannabinoid receptor (CB1) antagonist N-piperidino-5-(4-clorophenyl)-1-(2,4dichlorophenyl)-4-methyl-3-pyrazole-carboxamide (rimonabant, SR141716A $3 \mathrm{mg} \mathrm{kg}^{-1}$, i.p.) completely prevented the actions of HU210. The present results indicate that cannabinoids exert their antiepileptic effects by impeding pathological synchronization of neuronal networks in the hippocampus.

Keywords: CB1 receptor, multiple single-neuron recording, urethane, GABA

\section{INTRODUCTION}

The appearance of epileptic seizures is commonly associated with aberrant hypersynchronization of large neuronal aggregates (Walker and Kullmann, 1999). One of the most commonly affected brain areas is the hippocampus, where several mechanisms underlying pathological synchronization have been identified (Miles and Wong, 1983). Such mechanisms include alterations in gap junctional signaling (Jefferys, 1995), imbalances of excitatory/inhibitory currents (Cohen et al., 2002) and an enhancement of inhibitory GABAergic feedback (Haas et al., 1996). In the present study, hippocampal epileptiform activity was induced pharmacologically with kainic acid (KA). This excitotoxin produces an episode of status epilepticus (Sokal and Large, 2001) and complex epileptiform EEG patterns (Ben-Ari, 1985; Medvedev and Willoughby, 1999) when administered acutely in vivo.

Available evidence from human and animal seizure models suggests that various cannabinoid compounds (derived from the marijuana plant, Cannabis sativa) have antiepileptic properties (Cunha et al., 1980; Wallace et al., 2002). Furthermore, anandamide has been shown to modulate seizure threshold and severity through CB1 receptors implicating the endogenous cannabinoid system in epilepsy (Karanian et al., 2007). An earlier study by Hàjos et al. (2000) provided a mechanistic explanation for the effects of the endogenous cannabinoid receptor ligand anandamide. Using hippocampal slices, the authors showed that bath application of cannabinoid receptor agonists reduce the power of KA-induced network oscillations, these actions were prevented by pre-treatment with the CB1 receptor antagonist rimonabant.

Population studies provide valuable information in terms of temporal associations among recorded neurons and of specific features of single-neuronal firing. As functional synaptic correlations between the neurons within the population (activity correlations) are created, single-unit fire in phase, whereas spikes from outside the correlated network are out of phase (Abeles et al., 1993). The present study was aimed at investigating the effects of cannabinoid receptor activation on KA-induced bursting activity in the CA1 hippocampal layer of anesthetized rats. We employed multiple simultaneous extracellular single-neuron recordings to physiologically verify the antiepileptic actions of cannabinoids and to better delineate the dynamics of population discharge patterns underlying epilepsy.

\section{MATERIALS AND METHODS ANIMALS AND SURGICAL PROCEDURES}

Male Sprague-Dawley rats ( $n=13$, Charles River Laboratories), weighing $300-350 \mathrm{~g}$ at the time of surgery were housed in groups of three in large plastic cages and maintained on a 12:12-h lightdark cycle (lights on at 7:00 AM). Water and food were available ad libitum. All experiments were performed in accordance to UK Home Office regulations under project license 40/1955 and with NIH guidelines (publication 86-23). Animals were anesthetized with urethane (25\% w/v in water) at a dose of $1.4 \mathrm{~g} \mathrm{~kg}^{-1}$ (i.p.) and mounted on a modified Kopf 1730 stereotaxic apparatus.

\section{RECORDING PROCEDURES}

Recordings were made with a bundle of eight microwire electrodes (Formvar-coated stainless steel, $50-\mu \mathrm{m}$ diameter; NBLabs, TX, USA), with a stainless steel ground electrode connected to a unitygain 8 channel J-type field effect transistor headstage (NBLabs). Electrodes were stereotaxically positioned via fine micromanipulator control over the hippocampus $(-5.5 \mathrm{~mm} \mathrm{AP,} \mathrm{3.0} \mathrm{L} \mathrm{relative} \mathrm{to}$ bregma and $-2.2 \mathrm{DV}$ from the brain surface) according to Paxinos 
and Watson (1986). The temperature of the animal was maintained at $37.7^{\circ} \mathrm{C}$ with the aid of a rectal probe connected to a homeothermic control unit (Harvard Apparatus, MA, USA). The second level of amplification occurred at a 16-channel differential preamplifier (fixed gain $\times 100$; Plexon Inc, TX, USA) after which the signal was transferred to a Multi-Channel Acquisition Processor (Plexon Inc, TX, USA). This allowed for computer-controlled amplification, filtering, switching and digital signal processing of the signals originating from the microwires. The impedance of the electrodes was $\sim 300 \mathrm{k} \Omega$ measured at $1 \mathrm{kHz}$. Commercially available software (RASPUTIN; Plexon Inc, TX, USA) allowed on-line isolation and discrimination (using dual voltage-time widows or principal component analysis) of neuronal spikes from background noise. Typically, one to four action potential waveforms were observed and discriminated per microwire during any given experiment. Activity was also displayed on a Tektronix D11 5000 series dualbeam oscilloscope and corresponding action potential waveforms were isolated with the aid of a Gould 1425 Digital Oscilloscope and a Gould Type 125 waveform processor. Firing rate was also monitored aurally with the aid of a loudspeaker. Signals were filtered using a second order low cut-off $(500 \mathrm{~Hz})$ and high cut-off $(5 \mathrm{kHz})$, with a $50-\mathrm{Hz}$ notch filter. The gain block was a programmable gain multiplier with gain steps $1-30$ (total gain: $\times 1000$ to $\times 32,000$ ); typically the gain was set at $\times 10,000$ for the experiments.

\section{PHARMACOLOGICAL MANIPULATIONS}

HU210 was obtained from Tocris Cookson (UK, USA), KA was obtained from Sigma Chemical (UK, USA) and rimonabant (SR141716A) was a generous gift from Sanofi-Synthelabo. HU210 and rimonabant were resuspended to stock concentration $\left(10^{-2} \mathrm{M}\right)$ in ethanol and refrigerated at $-20^{\circ} \mathrm{C}$. Drugs were dissolved in a physiological $1.0-\mathrm{ml}$ saline $(0.9 \% \mathrm{NaCl}) / 10$ - $\mu \mathrm{l}$ Tween $80(1 \%)$ vehicle solution for injection on the day of the experiment. KA was resuspended to stock concentration $\left(10^{-2} \mathrm{M}\right)$ in distilled water and frozen $\left(-20^{\circ} \mathrm{C}\right)$ in $200-\mu$ l aliquots. When required, the aliquots were thawed and diluted in $0.2-\mathrm{ml}$ saline and for each experiment an aliquot was thawed and diluted in $0.2-\mathrm{ml}$ saline to the desired dose. All drugs were injected intraperitoneally (i.p.).

\section{ELECTROPHYSIOLOGICAL ANALYSES}

Discriminated single-unit data was initially visualized off-line as spike rasters; the spike raster representing action potential spikes, viewed as vertical ticks, corresponding to the temporal (time stamped) occurrence of the spikes. Data was subsequently analyzed as integrated firing rate histograms (accumulated over 5 or 10 s epochs); burst analyses were calculated for epochs of 15 s during periods of bursting activity and cross-correlation histograms (CCHs; Gerstein, 1970), between pairs of hippocampal neurons, were computed using NeuroExplorer (Plexon Inc, TX, USA). Basal activity was calculated as the mean \pm SEM of firing during a selected 10 -min pre-drug period for overall firing analyses and over $15 \mathrm{~s}$ for burst analyses. Visual evaluation of bursting discharge activity, induced by KA injection, in the spike raster displays revealed two types of neuronal discharge - "micro-bursts" and "macrobursts". Micro-bursts were empirically defined as follows: maximum burst start interval of $170 \mathrm{~ms}$, maximum interspike interval within the burst $300 \mathrm{~ms}$; minimum inter-burst interval $200 \mathrm{~ms}$; minimum duration for a burst $100 \mathrm{~ms}$ and minimum number of spikes within a burst, minimum of three spikes. Macro-bursts were much slower oscillations in overall firing (clearly visible over longer time frames - see Figure 3A) with a periodicity of 180-220 s. CCH analysis (Figure 1B) shows the conditional probability of the occurrence of a spike in the spike train at time $t$ on the condition that there is a reference event (or reference spike; in this case spikes from neuron 1) at time 0 (Gerstein, 1970). Therefore, if the graph shows a flat profile, neurons are not firing synchronously with respect to a given reference neuron. Correlated discharge activity (i.e., functional firing synchronicity) between pairs of neurons were revealed in CCHs as a central peak (or trough) centered on the zero time bin, while a flat cross-correlation indicated non-correlated discharge activity between neuronal pairs (Eblen-Zajjur and Sandkuhler, 1997). Conventional analyses of variance (ANOVA) were used to study treatment-response differences. The Scheffé's post-hoc test was used to assess differences between and within treatments. The criterion of significance for the ANOVAs and post-hoc tests was set at $p<0.05$. All data are expressed mean \pm SEM and statistical analyses were computed using Statistica (Statsoft Inc, OK, USA).

On completion of experiments, the location of the recording electrode tips was marked by passing a $60-\mu \mathrm{A}$ current for $30 \mathrm{~s}$ through electrode pairs in the array. Animals were then transcardially perfused with a $4 \%$ paraformaldehyde $/ 5 \%$ potassium ferrocyanide solution. Ferrocyanide creates a blue-green stain by reacting with deposited iron ions at the mark site. The brains were then removed and stored at $4^{\circ} \mathrm{C}$ in the same fixative solution overnight. Vibratome sections were then cut $(80 \mu \mathrm{m})$ and transferred to slides with the aid of mounting medium (Vectashield). Photographic images were made using a low power objective without further counter-staining of the sections.

\section{RESULTS}

A total of 58 neurons ( $n=6$ rats) were recorded showing action potential signal-to-noise amplitude ratios of at least a 3:1. KA $\left(10 \mathrm{mg} \mathrm{kg}^{-1}\right)$ produced a significant increase in the number of bursts over pre-drug activity in 46 of 58 units from $4.1 \pm 1.06$ to $10.5 \pm 1.18$ bursts (Figures 1A and 2; $F_{(1,57)}=15.74, p<0.0008$ ). Every neuron studied was significantly $(p<0.05)$ excited from predrug basal firing following KA injection (Figure 3; $6.89 \pm 1.18$ to $13.05 \pm 2.04 \mathrm{~Hz}$, for the entire population). The onset latency for KA observable effects was $\sim 6$ min after injection.

Inspection of firing rate histograms showed that KA treatment elicited two types of bursts: micro-bursts (as defined in "Materials and methods") and macro-bursts that lasted $~ 202 \pm 21.2 \mathrm{~s}$ in duration (Figure 3A). The number of KA-induced micro-burst events was significantly reduced by the high-affinity cannabinoid agonist HU210 from $10.5 \pm 1.18$ to $3.55 \pm 2.07$ bursts during each analysis period (Figures 1 and $\left.2 ; F_{(1,57)}=8.89, p<0.05\right)$. The KA-induced elevation in firing rate was only marginally reduced by concurrent administration of HU210 ( $\left.100 \mu \mathrm{g} \mathrm{kg}^{-1}\right)$, decreasing the firing rate of simultaneously recorded neurons from $13.05 \pm 2.04$ to $10.74 \pm 1.56 \mathrm{~Hz}$ on this short time scale $\left(F_{(2,85)}=1.1, p<0.38\right.$, data not shown). The frequency of macro-bursts was similarly inhibited (Figure 3) by HU210 from $3.7 \pm 1.1$ to $1.1 \pm 0.3$ bursts $\left(F_{(1.57)}=3.2, p<0.05\right.$, data not shown); however on this longer time scale, overall firing rate was significantly reduced $11.15 \pm 3.04$ to $4.84 \pm 2.26\left(F_{(2,85)}=8.4, p<0.05\right)$. 


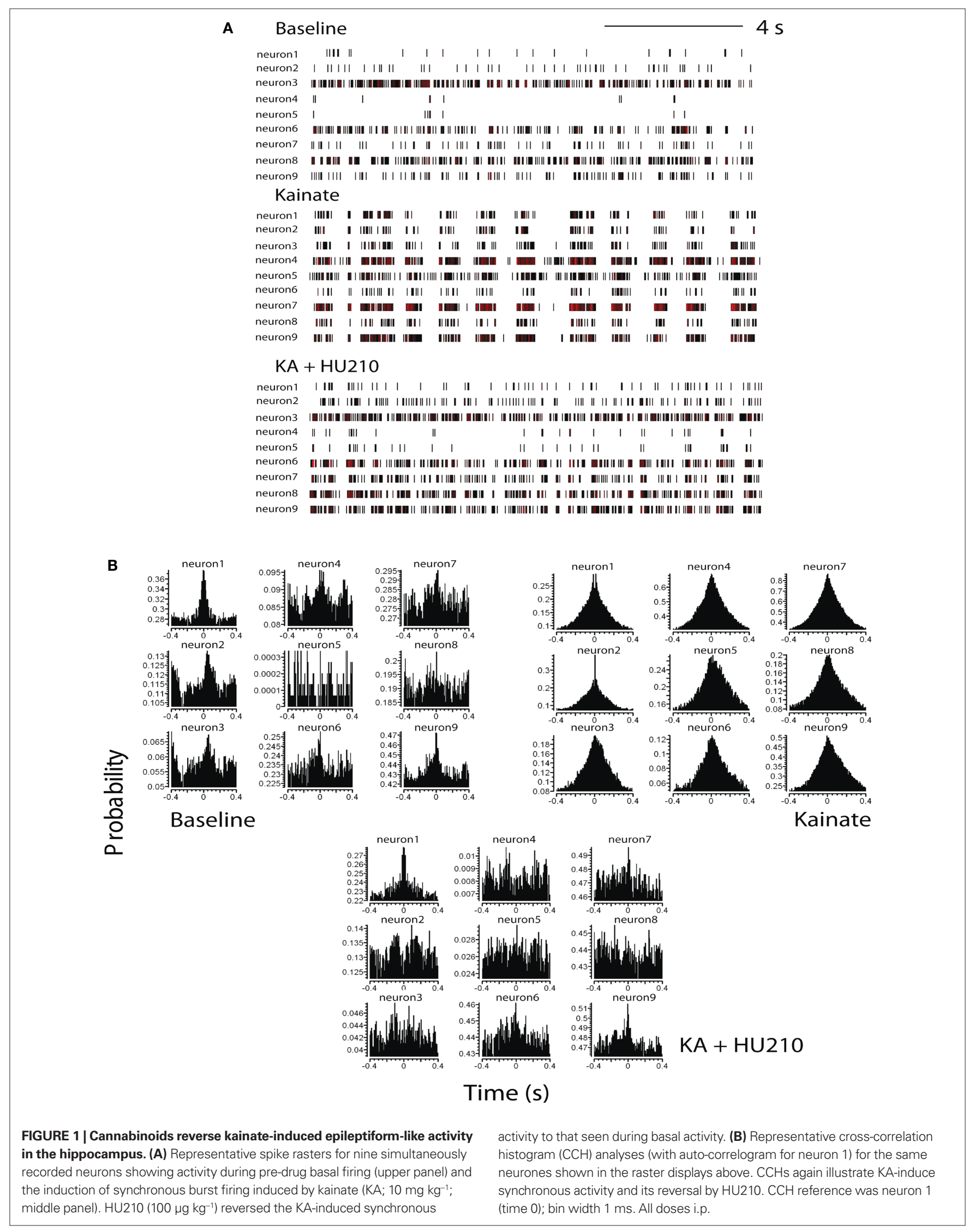




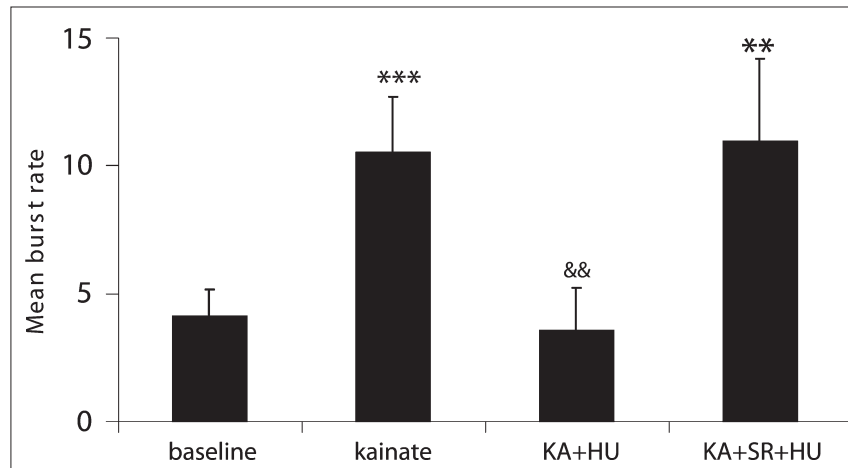

FIGURE 2 | Incidence of mean burst rate (bursts per analysis epoch \pm SEM) as a function of drug treatment. KA significantly increased the burst frequency from pre-drug basal activity and entrained the network to burst synchronously. Co-application of the CB1 receptor agonist HU210 (HU) significantly decreased burst rate and disrupted the synchronicity in firing induced by KA. When injected in the presence of the CB1 receptor antagonist rimonabant (SR), HU210 did not alter KA-induced synchronous bursting activity $\left({ }^{*} p<0.01 ;{ }^{* *} p<0.005\right.$ vs baseline and ${ }^{*} \& p<0.01$ vs KA-induced bursting)

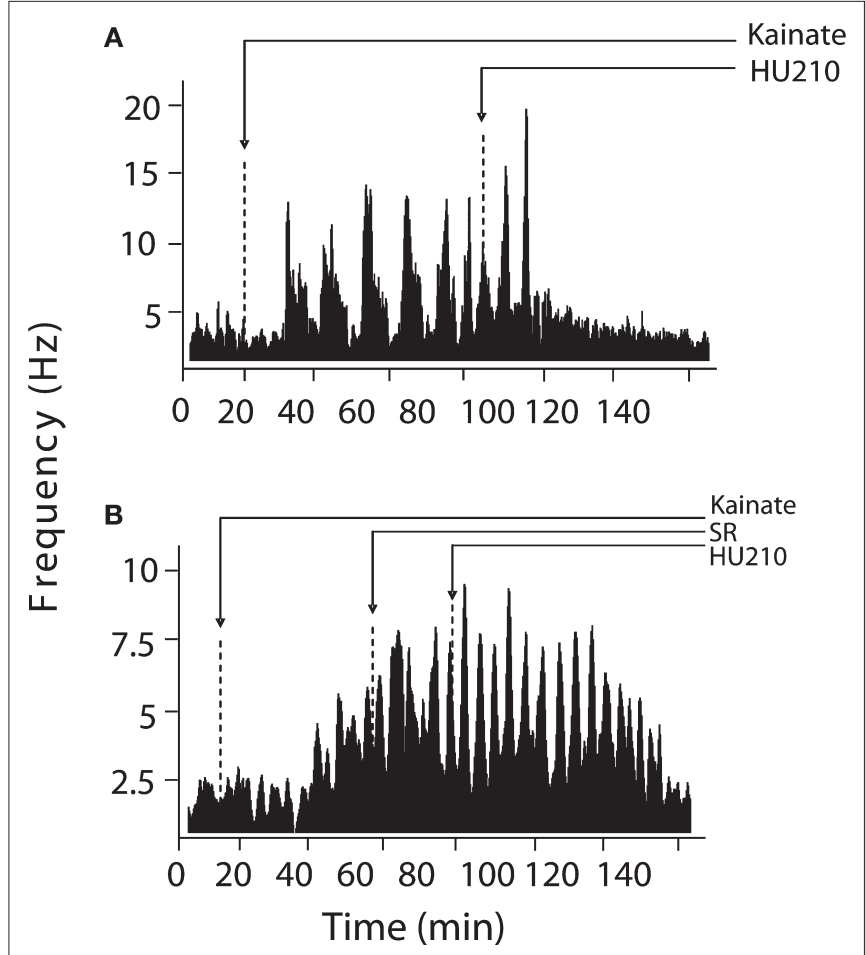

FIGURE 3 | Modulation of KA-induced slow oscillations in firing by CB1 receptors. (A) Representative integrated firing rate histograms (bin width $10 \mathrm{~s}$ ) illustrating KA-induced macro-burst firing pattern (periodicity $200 \mathrm{~s}$ ). Injection of HU210 abolished this slow oscillatory firing activity. (B) Pretreatment with the CB1 antagonist rimonabant (SR) completely blocked the actions of HU210. Arrow heads indicate the time of HU210 $\left(100 \mathrm{\mu g} \mathrm{kg}^{-1}\right)$ or rimonabant $\left(3 \mathrm{mg} \mathrm{kg}^{-1}\right)$ injections during $\mathrm{KA}\left(10 \mathrm{mg} \mathrm{kg}^{-1}\right)$. All doses i.p.

Cross-correlation histogram analyses confirmed the visual inspection of the raster displays that there is limited or no synchronous activity between hippocampal neurons during pre-drug basal activity, at least under the recording conditions of the present experiments (Figure 1B). Following KA injection, corresponding with the period of KA-induced micro-burst and macro-burst activity, there was development of synchronous activity, revealed by peaks in the CCHs around time bin 0 (Figure 1B). This was reversed by injection of $\mathrm{HU} 210$, resulting in $\mathrm{CCH}$ s showing a flat profile similar to that expressed under basal conditions (Figure 1B), indicative of non-synchronous activity.

The observed antiepileptic effects on hippocampal cell activity are mediated by $\mathrm{CB} 1$ receptors as the number of KA-induced microbursts was not significantly different from KA alone (9.98 \pm 3.21 ; $\left.F_{(2,77)}=4.3, p<0.69\right)$ when HU210 was injected in the presence of the $\mathrm{CB} 1$ receptor antagonist rimonabant (21 neurons, $n=3$ rats, $3 \mathrm{mg} \mathrm{kg}^{-1}$, Figure 2). Pre-treatment with rimonabant $\left(3 \mathrm{mg} \mathrm{kg}^{-1}\right)$ also prevented the blockade of KA-induced changes in firing rate and bursting activity patterns by HU210, whilst being devoid of effects of its own $(5.81 \pm 2.38 \mathrm{~Hz}$ during KA/SR and $7.01 \pm 3.13 \mathrm{~Hz}$ during KA/SR/HU210, and $9.1 \pm 1.13$ total bursts during KA/SR and $11.24 \pm 4.28$ total bursts during KA/SR/HU210, $F_{(2,77)}=8.1$, $p<0.34$, Figure 3 ).

\section{DISCUSSION}

The present in vivo multiple single-unit study has generated two main findings. First, functionally cross-correlated epileptiform-like population activity can be evoked by the non-NMDA receptor agonist KA in the hippocampal CA1 pyramidal layer of urethane-anesthetized rats, in a way reminiscent of epileptiformlike activity observed in hippocampal dissociated and organotypic cultures (Jagger et al., 2001, 2002; Roe and Mason, 2002; Sokal et al., 2000). The excitotoxin injection increased the firing rate of all the recorded neurons and also increased the burst frequency in the majority of simultaneously recorded cells whilst entraining them to a synchronized firing mode (no KA-induced inhibitions of firing were observed). Secondly, when injected in the presence of $\mathrm{KA}$, the potent $\mathrm{CB} 1$ receptor agonist HU210 significantly reduced the elevation in burst rate (accompanied by a modest reduction in overall firing rate) elicited by KA treatment accompanied by a reversal of synchronized burst firing to random activity.

The hippocampal formation is one of the most seizure-prone structures in the brain due to its defining characteristic; the presence of a tri-synaptic circuit of fibers. The reverberation of impulse activity in the hippocampal loop is suggested to cause and maintain epileptiform-like activity (Stringer and Lothman, 1992). Hippocampal cells are endowed with several voltage and ligand-gated conductances, which play a major role in the cells' excitability. Even under normal basal conditions cells produce burst discharges that are qualitatively similar to epileptiform-like activity. Blockade of GABAergic transmission has been consistently proven to precipitate seizures. It has thus been assumed that abnormal GABAergic neurotransmission may be related to epilepsy (Prince, 1978). Accordingly, the most common pharmacological manipulation to cause neuronal disinhibition is the use of the convulsant alkaloid bicuculline; a GABA ${ }_{A}$ receptor antagonist (Margineanu and Wülfert, 1999). However, in vivo epilepsy can also be induced with exposure to toxins or direct brain electrical stimulation, in a manner that may not be directly related to GABAergic mechanisms. One such agent is the excitotoxin KA, an AMPA/kainate receptor agonist. KA-induced seizures are a well-established model of temporal 
lobe epilepsy and epileptic seizures reliably occur at the chosen systemic dose of $10 \mathrm{mg} \mathrm{kg}^{-1}$ used in this study, a non-toxic dose (Sperk, 1994). Interestingly, Khazipov and Holmes (2003) elegantly implicated GABA inhibitory mechanisms in the emergence of synchronized epileptiform-like activity elicited by KA. In their in vivo superfused hippocampus preparation, GABA receptor-mediated currents coincided with the inhibition of neuronal firing as assessed by the cross-correlogram of spikes vs inhibitory postsynaptic currents. The authors found that when inhibitory currents ceased, the probability of firing of pyramidal cells increased. Mediation of $\mathrm{GABA}_{\mathrm{A}}$ receptors was further confirmed by the finding that bicuculline perfusion suppressed KA-induced oscillations.

The hippocampus is one of the areas with the highest cannabinoid receptor binding densities in the rat brain (Herkenham et al., 1991). This structure also exhibits the highest concentrations of both anandamide (Felder et al., 1996) and 2-arachidonylglycerol (Stella et al., 1997), the two most abundant endogenous cannabinoid ligands. It is now well accepted that presynaptic CB1 receptors are located on the terminals of cholecystokinin (CCK+)containing GABAergic interneurons to inhibit $\mathrm{Ca}^{2+}$ dependent GABA release via N-type channels (for review see Freund et al., 2003). Cannabinoid receptors are also reported to be situated on glutamatergic presynaptic terminals inhibiting glutamate release via terminal $\mathrm{N}$-type and $\mathrm{P} / \mathrm{Q}$ type $\mathrm{Ca}^{2+}$ channels (Twitchell et al., 1997) in autaptic circuits as well as in hippocampal slices (Kawamura et al., 2006). Thus, at least in the hippocampus, CB1 receptors are located on $(\mathrm{CCK}+)$ basket and dendritically projecting cells (Freund, 2003; Hàjos et al., 2000) as well as on excitatory afferents to pyramidal cells (Takahashi and Castillo, 2006). We are therefore left with the view that mixed actions of HU210 on inhibitory and excitatory neurotransmission are responsible for reversing synchronized burst firing. Since intact GABAergic inhibition is necessary for the maintenance of KA-induced bursting, the activation of $\mathrm{CB} 1$ receptors by $\mathrm{HU} 210$ on the terminals of different populations of GABAergic neurons in the hippocampus and the corresponding inhibition of $\mathrm{GABA}_{\mathrm{A}}$ synaptic inhibition (Hoffman and Lupica, 2000) could be sufficient to shunt synchronous population bursting. However, direct effects of CB1 agonists in suppressing glutamatergic excitatory neurotransmission have been observed in a KA model of seizure (Monory et al., 2006). This suggests CB1 receptors are also critically involved in the control of excitatory glutamatergic neurotransmission. Thus, activation of CB1 receptors may therefore confer inhibitory effects via suppression of excitatory synapses under hyperexcitable conditions. Nevertheless, because of the systemic nature of the dosing regimen employed in our study, contributions from outside the hippocampal circuitry cannot be ruled out (microinjection studies are currently ongoing in our laboratory to address this issue).

\section{REFERENCES}

Abeles, M., Bergman, H., Margalit, E., and Vaadia, E. (1993). Spatiotemporal firing patterns in the frontal cortex of behaving monkeys. J. Neurophysiol. 70, 1629-1638.

Ben-Ari, Y. (1985). Limbic seizure and brain damage produced by kainic acid: mechanisms and relevance to human temporal lobe epilepsy. Neuroscience 14, 375-403.

Cohen, I., Navarro, V., Clemenceau, S., Baulac, M., and Miles, R. (2002). On the origin of interictal activity in human temporal lobe epilepsy in vitro. Science 298, 1418-1421.

Coomber, B., O’Donoghue, M. F., and Mason, R. (2008). Inhibition of

The finding that the $\mathrm{CB} 1$ receptor antagonist rimonabant was devoid of effect on KA-induced synchronized bursting is puzzling (but see Robbe et al., 2006) since the present KA manipulations represent the ideal conditions for the on-demand release of endocannabinoids hippocampal slices. This may indicate that the basal level of endocannabinoid binding to CB1 receptors is not sufficient to effectively modulate epileptiform-like activity in anesthetized animals by virtue of achieving a ceiling effect wit the dose of KA used here. It would be interesting to determine whether an effect of rimonabant would be uncovered when recording are performed in awake, freely-moving rats. It would also be important to determine whether injection of rimonabant prevents the induction of epileptiform-like activity by KA, since it does not reverse KA effects on its own. Nevertheless, the present study replicates and builds upon previous findings that KA-induced increases in firing and burst firing rates are attenuated by inhibition of anandamide (an endocannabinoid) hydrolysis (Coomber et al., 2008). However, under those conditions rimonabant only partly diminished the effects of anandamide hydrolysis blockade (in that particular study functional connectivity was not assessed). The appearance of "macro-bursts" composed of "micro-bursts" in the present report could be associated with metabolic recovery following intense increases in firing rate. Alternatively, the extensive calcium influx during periods of sustained elevated activity may sequentially trigger downregulation of inhibitory and/or excitatory processing (see Hàjos and Freund, 2002) and the generation of the subsequent volley of intense firing.

These previously unseen findings suggest that the reduction in spike timing coordination and the associated decrease in hippocampal population activity are important in the well-documented antiepileptic actions of cannabinoid compounds (Smith, 2005). A major implication of these data is that the synchrony of spike timing in neuronal assemblies is a critical component of epileptiform-like activity in vivo. The effect of cannabinoids on network dynamics is noteworthy because neurons form assemblies defined by synchronous action potential firing and these changes in population synchronicity are thought to accompany status epilepticus (Williams et al., 2007).

In conclusion we have shown that cannabinoid receptors modulate the rhythmicity and synchronization generated by inhibitory neuronal networks in the rat hippocampus. Further investigations are required to determine the clinical relevance and the site of action of the cannabinoid agonist, since drugs that reduce synchronization may represent novel anticonvulsants.

\section{ACKNOWLEDGMENTS}

Thanks are due to Clare Spicer for technical support. This research was funded in part by NIH grant DA022340 to JFC.

endocannabinoid metabolism attenuates enhanced hippocampal neuronal activity induced by kainic acid. Synapse 62, 746-755.

Cunha, J. M., Carlini, E. A., Pereira, A. E., Ramos, O. L., Pimentel, C., Gagliardi, R., Sanvito, W. L., Lander, N., and Mechoulam, R. (1980). Chronic administration of cannabidiol to healthy volunteers and epileptic patients. Pharmacology 21, 175-185.

Eblen-Zajjur, A. A., and Sandkuhler, J. (1997). Synchronicity of nociceptive and non-nociceptive neurons in the spinal dorsal horn of the rat: stimulus-induced plasticity. Neuroscience $76,39-54$. 
Felder, C. C., Nielson, A., Briley, E. M., Palkovits, A., Priller, J., Axelrod, J. Richardson, J. M., Riggin, R. M., Paul, G. A., and Becker, G. W. (1996). Isolation and measurement of the endogenous cannabinoid receptor agonist, anandamide, in brain and peripheral tissues of human and rat. FEBS. Lett. 393, 231-235.

Freund, T.F. (2003). Interneuron diversity series: rhythm and mood in perisomatic inhibition. Trends Neurosci. 26, 489-495.

Freund, T. F., Katona, I., and Piomelli, D. (2003). Role of endogenous cannabinoids in synaptic signalling. Physiol. Rev. 83, 1017-1066.

Gerstein, G. L. (1970). Functional association of neurones: detection and interpretation. In The Neurosciences: Second Study Program, F. O. Schmitt, ed. (New York, NY, The Rockefeller University Press), pp. 648-661.

Haas, K. Z., Sperber, E. F., Moshé, S. L., and Stanton, P. K. (1996). Kainic acidinduced seizures enhance dentate gyrus inhibition by down-regulation of $\mathrm{GABA}_{\mathrm{B}}$ receptors. J. Neurosci. 16, 4250-4260.

Hàjos, N., and Freund, T. F. (2002). Pharmacological separation of cannabinoid sensitive receptors on hippocampal excitatory and inhibitory fibres. Neuropharmacology 43 , 503-510.

Hàjos, N., Katona, I., Naiem, S. S., MacKie, K., Ledent, C., Mody, I., and Freund, T. F. (2000). Cannabinoids inhibit hippocampal GABAergic transmission and network oscillations. Eur. J. Neurosci. 9, 3239-3249.

Herkenham, M., Lynn, A. B., Johnson, M. R., Melvin, L. S., de Costa, B. R., and Rice, K. C. (1991). Characterisation and localisation of cannabinoid receptors in rat brain: a quantitative in vitro autoradiographic study. J. Neurosci. 11, 563-583.

Hoffman, A. F., and Lupica, C. R. (2000). Mechanisms of cannabinoid inhibition of GABA(A) synaptic transmission in the hippocampus. J. Neurosci. 20, 2470-2479.
Jagger, E., Parker, L., Hope, D. T., and Mason, R. (2001). Effect of magnesium ions on bicuculline-induced epileptiform activity in hippocampal organotypic slices and monolayer hippocampal neuronal networks. $B r$. J. Pharmacol. 134, 126.

Jagger, E., Parker, T. L., Starkey, S., and Mason, R. (2002). Kainic acid-induced epileptiform activity and cell death in hippocampal organotypic cultures. Br. J. Pharmacol. 135, 103.

Jefferys, J. G. (1995). Non-synaptic modulation of neuronal activity in the brain: electric currents and extracellular ions. Physiol. Rev. 75, 689-723.

Karanian, D. A., Karim, S. L., Wood, J. T., Williams, J. S., Lin, S., Makriyannis, A., andBahr,B.A.(2007).Endocannabinoid enhancement protects against kainic acid-induced seizures and associated brain damage. J. Pharmacol. Exp. Ther. 322, 1059-1066.

Kawamura, Y., Fukaya, M., Maejima, T., Yoshida, T., Miura, E., Watanabe, M., Ohno-Shosaku, T., and Kano, M. (2006). The CB1 cannabinoid receptor is the major cannabinoid receptor at excitatory presynaptic sites in the hippocampus and cerebellum. J. Neurosci. 26, 2991-3001.

Khazipov, R., and Holmes, G. L. (2003). Synchronisation of kainate-induced epileptic activity via GABAergic inhibition in the superfused rat hippocampus in vivo. J. Neurosci. 23, 5337-5341.

Margineanu, D. G., and Wülfert,E. (1999). Differential paired-pulse effects of gabazine and bicuculline in rat hippocampal CA3 area. Brain Res. Bull. 51, 69-74.

Medvedev, A., and Willoughby, J. O. (1999). Autoregressive modelling of the EEG in systemic kainic acid-induced epileptogenesis. Int. J. Neurosci. 97, 149-167.

Miles, R., and Wong, R. K. S. (1983). Single neurones can initiate synchronised population discharge in the hippocampus. Nature 306, 371-373.

Monory, K., Massa, F., Egertová, M., Eder, M.,Blaudzun,H.,Westenbroek, R.,
Kelsch, W., Jacob, W., Marsch, R., Ekker, M., Long, J., Rubenstein, J. L., Goebbels, S., Nave, K. A., During, M. Klugmann, M.,Wölfel, B., Dodt, H. U., Zieglgänsberger, W., Wotjak, C. T. Mackie, K., Elphick, M. R. Marsicano, G., and Lutz, B. (2006). The endocannabinoid system controls key epileptogenic circuits in the hippocampus. Neuron 51, 455-466.

Paxinos, G., and Watson, C. (1986). The Rat Brain in Stereotaxic Co-ordinates, 2nd Edn. New York, NY, Academic Press.

Prince, D. A. (1978). Neurophysiology of epilepsy. Annu. Rev. Neurosci. 1, 359-415.

Robbe, D., Montgomery, S. M., Thome, A., Rueda-Orozco, P. E., McNaughton, B. L., and Buzsaki, G. (2006). Cannabinoids reveal importance of spike timing coordination in hippocampal function. Nat Neurosci. 9, 1526-1533.

Roe, C., and Mason, R. (2002). Effects of cannabinoid (CB) agonists on rat hippocampal neuronal firing rate activity using multi-channel extracellular recording. Br. J. Pharmacol. 137, 109.

Smith, P. F. (2005). Cannabinoids as potential anti-epileptic drugs. Curr. Opin. Investig. Drugs 6, 680-685.

Sokal, D. M., and Large, C. H. (2001). The effects of GABA(B) receptor activation on spontaneous and evoked activity in the dentate gyrus of kainic acidtreated rats. Neuropharmacology 40, 193-202.

Sokal, D. M., Mason, R., and Parker, T. L. (2000). Multi-neuronal recordings reveal a differential effect of thapsigargin on bicuculline- or gabazine-induced epileptiform excitability in rat hippocampal neuronal networks. Neuropharmacology 39 2408-2417.

Sperk, G. (1994). Kainic acid seizures in the rat. Prog. Neurobiol. 42, 1-32.

Stella, N., Schweitzer, P., and Piomelli, D. (1997). A second endogenous cannabinoid that modulates long-term potentiation. Nature 388, 773-778.
Stringer, J. L., and Lothman, E. W. (1992). Reverberatory seizure discharges in hippocampal-parahippocampal circuits. Exp. Neurol. 116, 198-203.

Takahashi, K. A., and Castillo, P.E. (2006). The CB1 cannabinoid receptor mediates glutamatergic synaptic suppression in the hippocampus. Neuroscience 139, 795-802.

Twitchell, W., Brown, S., and Mackie, K. (1997). Cannabinoids inhibit N- and P/Q-type calcium channels in cultured rat hippocampal neurones. J. Neurophysiol. 78, 43-50.

Walker, M. C., and Kullmann, D. M (1999). Febrile convulsions: a"benign" condition? Nat. Med. 5, 871-872.

Wallace, M. J., Martin, B. R., and DeLorenzo, R. J. (2002). Evidence for a physiological role of endocannabinoids in the modulation of seizure threshold and severity. Eur. J. Pharmacol. 452, 295-301.

Williams, P. A., Hellier, J. L., White, A. M. Staley, K. J., and Dudek, F. E. (2007). Development of spontaneous seizures after experimental status epilepticus: implications for understanding epileptogenesis. Epilepsia 48, 157-163.

Conflict of Interest Statement: The authors declare that the research was conducted in the absence of any commercial or financial relationships that could be construed as a potential conflict of interest.

Received: 20 February 2009; paperpending published: 12 May 2009; accepted: 02 June 2009; published online: 15 June 2009.

Citation: Mason R and Cheer JF (2009) Cannabinoid receptor activation reverses kainate-induced synchronized population burst firing in rat hippocampus. Front. Integr. Neurosci. (2009) 3:13. doi:10.3389/neuro.07.013.2009 Copyright $\odot 2009$ Mason and Cheer. This is an open-access article subject to an exclusive license agreement between the authors and the Frontiers Research Foundation, which permits unrestricted use, distribution, and reproduction in any medium, provided the original authors and source are credited. 